\title{
Relationship between mean platelet volume, low-grade systemic coagulation and vitamin D deficiency in canine visceral leishmaniasis
}

\author{
HASAN ERDOGAN, KEREM URAL, SERDAR PASA
}

Department of Internal Medicine, Faculty of Veterinary Medicine, University of Adnan Menderes, Aydın, Turkey

\section{Erdogan H., Ural K., Pasa S.}

\section{Relationship between mean platelet volume, low-grade systemic coagulation and vitamin D deficiency in canine visceral leishmaniasis}

\section{Summary}

Canine visceral leishmaniasis is associated with cardiac changes. This study was designed to test the hypothesis that vitamin D and coagulation parameters, such as D-dimer, activated partial thromboplastin time (APTT), partial thromboplastin time (PT), mean platelet volume (MPV), and white blood cell (WBC) levels, change in different stages of canine visceral leishmaniasis (CVL). Thirty-two dogs diagnosed with CVL were classified into four different groups: stage I (mild disease), stage II (moderate disease), stage III (severe disease), stage IV (very severe disease), and healthy controls. The groups were based on clinical signs, rapid ELISA/IFAT, hematological and serum biochemical tests, and urinary protein/creatinine ratios. Serum vitamin D levels were positively correlated with MPV $(r=0.503)$, but negatively correlated with $D$-dimer $(r=-0.326)$, APTT $(r=-0.361)$, PT $(r=-0.289)$, and $\mathrm{WBC}(\mathrm{r}=-\mathbf{0 . 3 8 4})$. The dogs with leishmaniasis showed increased WBC levels compared with the control group. Similarly, their vitamin D levels were significantly decreased compared with those in the control group $(\mathrm{p}<\mathbf{0 . 0 5})$. Severely diseased dogs (stage IV leishmaniasis) showed the lowest vitamin D levels, but there were no significant differences between dogs in the various stages of leishmaniasis. The evidence provided by this study indicates that the CVL dogs had low-grade systemic coagulation and fibrinolytic activation, as indicated by elevated MPV, PT, WBC and D-dimer levels, which may be used as a biomarker of low-grade systemic inflammation in CVL.

Keywords: canine visceral leishmaniasis, coagulation, low grade, stage

Canine visceral leishmaniasis (CVL), a well-known parasitic and zoonotic disease caused by an intracellular protozoan of the genus Leishmania (22), is transmitted to canines by the bite of blood-sucking sand flies (Phlebotomus species). The zoonotic potential means that the risk of undiagnosed, unmanaged leishmaniasis increases significantly in many parts of the world (1, $8,15,19)$. The disease might be present subclinically or manifest itself as systemic symptoms (cutaneous, ocular or renal lesions, hepatosplenomegaly, anemia, muscular atrophy and weight loss) (9). However, at a severe stage of the disease, altered hemostasis predisposes infected dogs to bleeding. There is limited research into the relationship between platelet function and leishmaniasis $(5,12,21,31)$. Various reports demonstrate that fibrinolysis leads to either hypocoagulability, a characteristic increase in some coagulation parameters (APTT, PT and D-dimer), or compensated DIC, with normal fibrinogen levels in CVL $(5,12,21,31)$.

Prior studies have demonstrated that cardiac disorders are linked to leishmaniasis $(6,9,26,32)$. Furthermore, the protozoan might be identified in cardiac tissue $(22,26)$. In medicine, several studies have demonstrated an association between cardiovascular mortality/morbidity and vitamin D deficiency. Low vitamin D levels are related to inflammation, impaired endothelial function, development of coronary artery calcium scores, and increased vascular stiffness (18, $28,30)$. Changes in platelet morphology and functions have been found in patients diagnosed with stroke, metabolic syndrome, and bleeding tendency. Recent studies have shown that MPV can be a useful biomarker for future myocardial infarctions and thromboembolism $(2,11)$. In addition, new data on vitamin $\mathrm{D}$ indicates that it might play a critical role in 
preventing platelet activation, along with fibrinolysis and thrombosis. This suggests that vitamin D insufficiency leads to increased mean platelet values (17). Therefore, the goal of this study, performed on dogs naturally exposed to different stages of CVL, was to determine the relationship between the mean platelet volume, low-grade systemic coagulation and vitamin D deficiency.

\section{Material and methods}

Location of the research and the dogs involved. The research was carried out at the University of Adnan Menderes, Faculty of Veterinary Medicine, Department of Internal Medicine in Aydin City, Turkey. It involved subjects with CVL and subjects without CVL.

Staging and classification of dogs. Prior to enrollment, ethical guidelines were established, and written owner consent was obtained. A total of 40 dogs were enrolled, dogs diagnosed with CVL showed clinical findings and were forwarded to a point-of-care ELISA test kits and immune fluorescence antibody testing. Dogs differentially diagnosed with CVL through clinical, serological, hematological, biochemical and urinalysis were classified into four different groups $(n=8)$, as previously reported by the Leishvet Group (29). The study groups were classified as Group A, Stage I (mild disease); Group B, Stage II (moderate disease); Group C, Stage III (severe disease); Group D, Stage IV (very severe disease); and Group E, healthy controls. Laboratory analyses that determined the disease stages are shown in Table 1.

Hematological examination. Blood specimens $(2 \mathrm{~mL}$ anticoagulated with EDTA) were drawn from $V$. cephalica antebrachia and forwarded to the laboratory. Complete blood counts, including leukocyte (WBC), packed cell volume (PCV), erythrocyte (RBC), hemoglobin (HGB), mean erythrocyte hemoglobin concentration (MCHC), mean erythrocyte volume (MCV), and thrombocyte (PLT) parameters, were analyzed with a cell counter (Abacus Junior).

Urinalysis. Drained urine samples or urine collected by catheterization $(5 \mathrm{~mL})$ were examined for total protein and creatinine concentrations in a private [Bio-Rad Eqas, Vetqas (Vet. Laboratory Quality Assurance Unit) and Vqas (Vet. Laboratory Quality Association Assurance Programme)] Veterinary Diagnosis and Analysis Laboratory, Istanbul, Turkey), in which urinary protein/creatinine ratio was calincluding 32 animals with CVL and 8 healthy ones. The outer quality program partnership laboratory (Bilim Special

culated colorimetrically. All urinary samples were immediately put in cold storage.

Serology-based assays. ELISA-based assay kits were used according to the manufacturer's guidelines to detect Leishmania infantum (Snap Leishmania test kit, IDEXX). An immunofluorescence assay $(5,29)$ was used to test sera samples for antibodies against Leishmania infantum (Leishmania IgG), as already described (29). Serum specimens for serological assays were stored until analysis. The IFAT result was considered positive for CVL with a $1: 64$ and higher titration of the serum that presented a clear yellow-green fluorescent signal in microscopic examination, whereas samples without a yellow-green fluorescent appearance were considered negative (29).

Serum biochemical analysis. Total protein, albumin and creatinine levels were determined by a chemistry analyzer (Samsung Lab Geo PT10, Korea).

Statistical analysis. Statistical analysis of the results was performed by SPSS 15.0. Arithmetic mean $(\overline{\mathrm{x}})$ and standard error values were calculated for all groups. All sets of data were examined for homogeneity by the Shapiro-Wilk analysis and were found normal. Comparisons of parameters in the groups were performed by analyses of variance (ANOVA), and the correlations between the levels of vitamin $\mathrm{D}$ and other parameters were determined. A $p<0.05$ was considered statistically significant.

\section{Results and discussion}

Significant differences in vitamin D, coagulation parameters, such as APTT, PT, and D-dimer, and other relevant biomarkers, such as WBC and MPV, were noted between the groups $(p<0.01)$. APTT, PT, $\mathrm{D}$-dimer, WBC and MPV values were higher in the diseased dogs than they were in the healthy subjects, whereas vitamin D levels were significantly lower. Cross-correlation analyses for vitamin D and other relevant parameters were performed. Serum vitamin D levels were positively correlated with MPV $(r=0.503$, $\mathrm{p}=0.002)$ and negatively correlated with D-dimer $(\mathrm{r}=-0.326, \mathrm{p}=0.056)$, APTT $(\mathrm{r}=-0.361, \mathrm{p}=0.033)$, PT $(r=-0.289, p=0.092)$ and WBC $(r=-0.384$ $\mathrm{p}=0.023)$.

The correlation between serum vitamin $\mathrm{D}$ levels and serum WBC, MPV, D-dimer, APTT and PT levels was positive for MPV $(r=0.503, p=0.002)$ and negative for D-dimer $(r=-0.326, p=0.056)$, APTT $(r=-0.361, p=$

Tab. 1. Biochemical parameters for the leishmaniatic groups and the control group (mean \pm SD)

\begin{tabular}{|c|c|c|c|c|c|}
\hline \multirow{2}{*}{ Parameters } & \multicolumn{4}{|c|}{ Stage } & \multirow{2}{*}{ Control } \\
\hline & I & II & III & IV & \\
\hline D-dimer & $1.0 \pm 0.77^{a}$ & $1.06 \pm 0.85^{a}$ & $3.91 \pm 2.36^{b}$ & $5.30 \pm 2.07^{b}$ & $0.1 \pm 0.0^{a}$ \\
\hline APTT & $12.90 \pm 1.42^{\mathrm{a}}$ & $13.15 \pm 2.27^{a}$ & $16.41 \pm 3.62^{b}$ & $17.99 \pm 2.75^{b}$ & $11.34 \pm 0.41^{a}$ \\
\hline PT & $7.20 \pm 0.27^{\mathrm{a}}$ & $7.38 \pm 1.61^{\mathrm{a}}$ & $9.32 \pm 1.18^{b}$ & $13.99 \pm 3.21^{\mathrm{b}}$ & $7.12 \pm 0.34^{\mathrm{a}}$ \\
\hline WBC & $14.92 \pm 4.36^{\mathrm{a}}$ & $13.92 \pm 3.98^{\mathrm{a}}$ & $14.88 \pm 6.06^{\mathrm{a}}$ & $19.74 \pm 13.50^{\mathrm{a}}$ & $6.74 \pm 1.10^{b}$ \\
\hline Vitamin D & $21.05 \pm 9.30^{a}$ & $20.70 \pm 8.34^{a}$ & $30.99 \pm 15.36^{a}$ & $16.37 \pm 4.89^{a}$ & $79.27 \pm 24.94^{b}$ \\
\hline MPV & $9.10 \pm 0.59^{a}$ & $8.86 \pm 1.30^{a b}$ & $10.16 \pm 1.73^{a}$ & $10.70 \pm 1.44^{\mathrm{a}}$ & $7.59 \pm 0.57^{\mathrm{b}}$ \\
\hline
\end{tabular}

Explanations: ${ }^{\mathrm{a}, \mathrm{b}}$ Different letters mark statistically significant differences between groups at $\mathrm{p} \leq 0.05$
$0.033), \mathrm{PT}(\mathrm{r}=-0.289$, $(\mathrm{r}=-0.384, \mathrm{p}=0.023)$ (Tab. 2). The correlations are shown in a scatter graph (Fig. 1).

WBC levels in the leishmaniotic groups were elevated, and there was a statistical significance between the control group and $\mathrm{p}=0.092)$, and WBC 

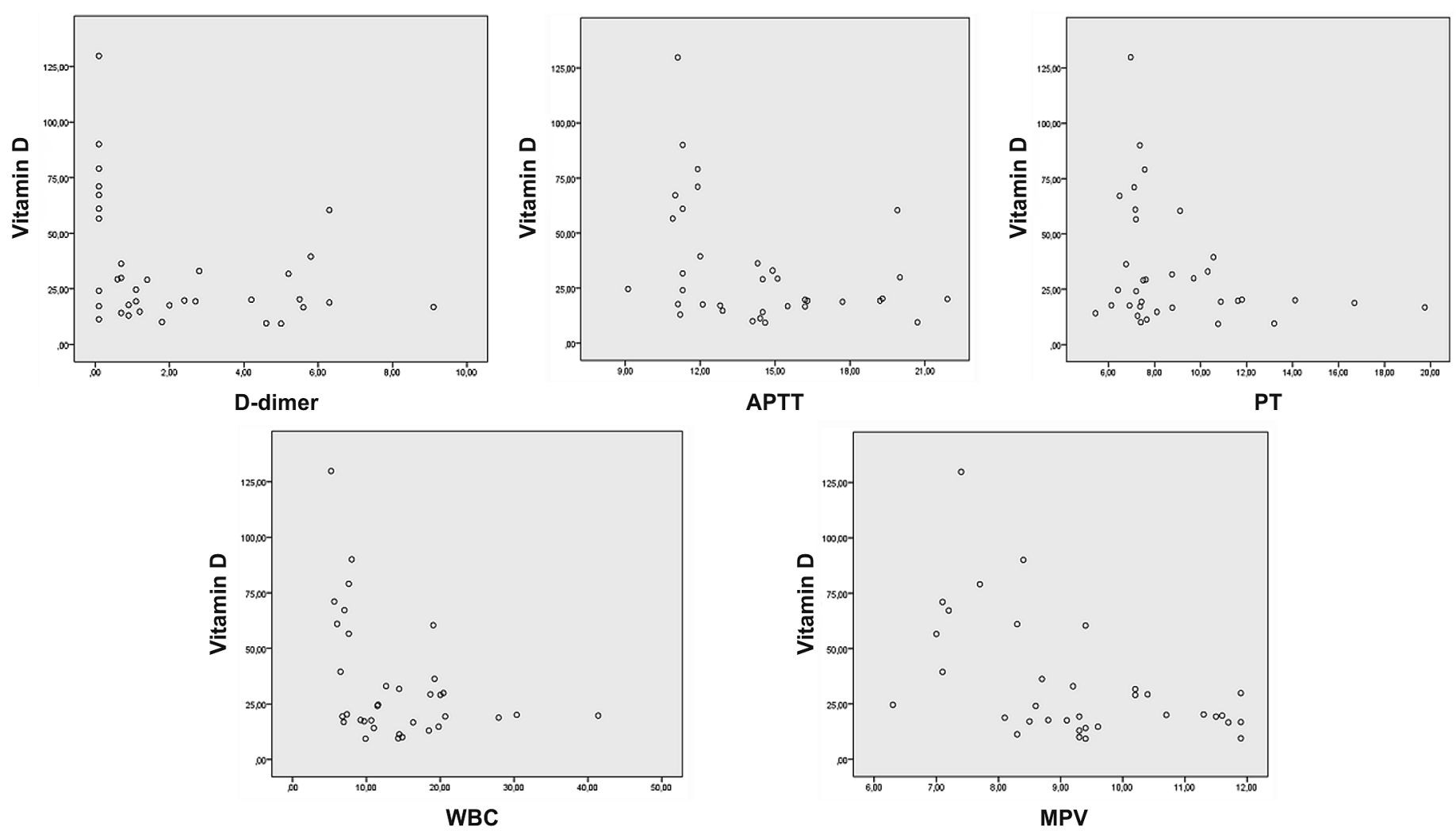

Fig. 1. Scatter graph showing correlations between serum vitamin D and serum WBC, MPV, D-dimer, APTT and PT levels

Tab. 2. Correlations between serum vitamin D levels and serum WBC, MPV, D-dimer, APTT and PT levels

\begin{tabular}{|l|c|c|c|c|c|}
\hline & D-dimer & APTT & PT & WBC & MPV \\
\hline \multirow{2}{*}{ Vitamin D } & $\mathrm{r}=-\mathbf{0 . 3 2 6}$ & $\mathrm{r}=-\mathbf{0 . 3 6 1}$ & $\mathrm{r}=-\mathbf{0 . 2 8 9}$ & $\mathrm{r}=-0.384$ & $\mathrm{r}=\mathbf{0 . 5 0 3}$ \\
& $\mathrm{p}=\mathbf{0 . 0 5 6}$ & $\mathrm{p}=\mathbf{0 . 0 3 3}$ & $\mathrm{p}=\mathbf{0 . 0 9 2}$ & $\mathrm{p}=\mathbf{0 . 0 2 3}$ & $\mathrm{p}=\mathbf{0 . 0 0 2}$ \\
\hline
\end{tabular}

Given the data demonstrating that vitamin D elevates antimicrobial peptides (27), the absence of vitamin D3 mediates inhibition of macrophages and microbicidal activity in vitamin $D$ receptors in knock-out mice, so the absence of vitathe leishmaniotic groups $(\mathrm{p}<0.05)$. By contrast, mean WBC values did not differ significantly between the diseased dogs. Similarly, vitamin D levels were significantly decreased compared to those in the control group $(\mathrm{p}<0.05)$, but there were no differences between the diseased dogs, which was an expected finding. The lowest vitamin D levels were found in severely diseased leishmaniotic dogs (stage IV). D-dimer levels in stage III and IV dogs were statistically significantly different from those in stage I and II dogs and to those in the healthy control dogs. APTT levels were also increased in stage III and stage IV dogs, but no significant change was found in stage I and II dogs. MPV levels, interestingly, showed insignificant differences between the diseased groups, but presented significance between control group.

In the current study, vitamin D deficiency was significantly more prevalent in the diseased dogs than it was in the healthy control group dogs. In view of the role of vitamin D in the control of leishmania infections, this vitamin has been recognized as an important element in the maintenance of homeostasis (25). Although the influence of vitamin D has been extensively researched, little data exists regarding its impact on parasitic diseases, especially leishmaniasis. min D results in elevated resistance to leishmaniasis (10). To our knowledge, different stages of CVL have not been sufficiently described. Inadequate vitamin D3 levels detected in the present study in dogs with canine visceral leishmaniasis highlight the need to improve the understanding of vitamin D supplementation and deficiency in future control studies. The vitamin D status of the host may influence the immune response to infections for which vitamin D deficiency appears to induce Th2-type cytokines profile (4). As previously determined by the LeishVet guidelines, Th2 is the predominant response, along with high serology, decreased cellular immunity, and high parasitic load (29). Vitamin D has been shown to increase Th2 cytokine levels $(3,4)$ and to reduce Th1 levels $(3,7,20)$. On the other hand, another study found that vitamin $D$ inhibited Th1 levels without changing Th2 levels (23). All suggested and proposed mechanisms might be responsible for differences in vitamin $\mathrm{D}$ levels between the diseased and control groups in the present study.

In the present study, we found a significant positive correlation between vitamin D and MPV $(r=0.503$, $\mathrm{p}=0.002)$ and negative correlation between vitamin $\mathrm{D}$ and WBC $(\mathrm{r}=-0.384, \mathrm{p}=0.023)$, PT $(\mathrm{r}=-0.289$, $\mathrm{p}=0.092)$, D-Dimer $(\mathrm{r}=-0.326, \mathrm{p}=0.056)$, and 
APTT $(r=-0.361, p=0.033)$. D-dimer levels were elevated in every advanced stage of the disease (exactly four-fold and five-fold increases in stages III and IV, respectively). This might be briefly explained by active and excessive blood coagulation (16). Another possible reason might be vasculitis (stage III, severe disease) or pulmonary thromboembolism (stage IV, very severe disease), which probably occurred in connection with canine visceral leishmaniasis (29).

Our study also demonstrated elevated MPV levels in leishmaniatic dogs and revealed a positive correlation between MPV and vitamin D levels. However, changes in mean MPV values were not statistically significant in dogs infected with Ehrlichia canis and Anaplasma phgocytophilium (24). In this study, dogs infected with leishmaniasis presented increased MPV values, which suggests that larger platelets play an active role in homeostasis $(13,14)$.

A limitation of this study is that a small number of CVL infected dogs were enrolled. However, the stages of the disease showed unexpected results. Furthermore, the study was designed to assess differences in the mean levels of vitamin D, PT, D-dimer, APTT, WBC and MPV, and significant differences were found. Lowgrade systemic inflammation revealed by vitamin $\mathrm{D}$, PT, D-dimer, APTT, WBC and MPV levels, similar to what has been shown elsewhere in women with primary ovarian insufficiency (16), should be taken into consideration in canine visceral leishmaniasis. There is a need for further studies of larger dog populations with CVL, describing different stages of the disease, as was the case in this study.

\section{References}

1.Al-Bajalan M. M., Niranji S. S., Al-Ja S. M., Kato H.: First identification of L. major in a dog in an endemic area of human cutaneous leishmaniasis in Iraq: molecular and phylogenetic studies. J. Parasitol. Res. 2018, 117, 585-590.

2.Bozkurt N., Yilmaz E., Biri A., Taner Z., Himmetoğlu O.: The mean platelet volume in gestational diabetes. J. Thromb. Thrombolysis 2006, 22, 51-54.

3. Cantorna M. T., Humpal-Winter J., DeLuca H. F.: In vivo upregulation of interleukin-4 is one mechanism underlying the immunoregulatory effects of 1, 25-dihydroxyvitamin D3. Arch. Biochem. Biophys. 2000, 377, 135-138.

4. Cantorna M. T., Zhu Y., Froicu M., Wittke A.: Vitamin D status, 1, 25-dihydroxyvitamin D3, and the immune system. Am. J. Clin. Nutr. 2004, 80, 1717-1720.

5. Ciaramella P., Pelagalli A., Cortese L., Pero M. E., Corona M., Lombardi P., Persechino A.: Altered platelet aggregation and coagulation disorders related to clinical findings in 30 dogs naturally infected by Leishmania infantum. Vet. J. 2005, 169, 465-467.

6. Costagliola A., Piegar G., Otrocka-Domagala I., Ciccarelli D., Iovane V., Oliva G., Paciello O.: Immunopathological features of canine myocarditis associated with Leishmania infantum infection. Biomed. Res. Int. 2016, 6.

7. D'ambrosio D., Cippitelli M., Cocciolo M. G., Mazzeo D., Di Lucia P., Lang R., Panina-Bordignon P.: Inhibition of IL-12 production by 1, 25-dihydroxyvitamin D3. Involvement of NF-kappaB downregulation in transcriptional repression of the p40 gene. J. Clin. Invest. 1998, 10, 252-262.

8. Dandrieux J. R. S., Sacchini F., Harms G., Globokar M., Balzer H. J., Pantchev N.: Canine Leishmania infantum infection: an imported case in UK after staying in the Canary Islands. J. Parasitol. Res. 2018, 117, 331-334.

9. Dos Santos F. P., Pascon J., Pereira D., Anjos B. L., Mistieri M., Silveira I. D., Porciuncula M. L.: Clinical and histopathological features of myocarditis in dogs with visceral leishmaniasis. Arq. Bras. Med. Vet. Zootec. 2015, 67, 1519-1527.
10. Ehrchen J., Helming L., Varga G., Pasche B., Loser K., Gunzer M., Lengeling A.: Vitamin D receptor signaling contributes to susceptibility to infection with Leishmania major. FASEB J. 2007, 21, 3208-3218.

11. Erikçi A. A., Muhçu M., Dündar O., Oztürk A.: Could mean platelet volume be a predictive marker for gestational diabetes mellitus? Hematol. 2008, 13, 46-48.

12. Font A., Closa J. M., Molina A., Mascort J.: Thrombosis and nephritic syndrome in a dog with visceral leishmaniasis. J. Small. Anim. Pract. 1993, 34, 466-470.

13. Gillum R. F., Mussolino M. E., Madans J. H.: Counts of neutrophils, lymphocytes, and monocytes cause specific mortality and coronary heart disease: the NHANES-I epidemiologic follow-up study. Ann. Epidemiol. 2005, 15, 266-271

14. Jakubowski J. A., Thompson C. B., Vaillancourt R., Valeri C. R., Deykin D.: Arachidonic acid metabolism by platelets of differing size. Br. J. Haematol. 1983, 53, 503-511.

15. Kaabi B., Zhioua E.: Modeling and comparative study of the spread of zoonotic visceral leishmaniasis from Northern to Central Tunisia. Acta Trop. 2018,178, 19-26.

16. Kebapcilar A. G., Kulaksizoglu M., Ipekci S. H., Korkmaz H., Kebapcilar L., Akyurek F., Gone M. S.: Relationship between mean platelet volume and low-grade systemic coagulation with vitamin D deficiency in primary ovarian insufficiency. Arch. Gynecol. Obstet. 2013, 288, 207-212.

17. Koyama T., Hirosawa S.: Anticoagulant effects of synthetic retinoids and activated vitamin D3. Semin. Thromb. Hemost. 1998, 24, 217-226.

18. Kunadian V., Ford G. A., Bawamia B., Qiu W., Manson J. E.: Vitamin D deficiency and coronary artery disease: a review of the evidence. Am. Heart. J. 2014, 167, 283-291.

19. Le Rutte E. A., van Straten R., Overgaauw P. A.: Awareness and control of canine leishmaniosis: A survey among Spanish and French veterinarians. Vet. Parasitol. 2018, 253, 87-93.

20. Lemire J. M.: Immunomodulatory actions of 1, 25-dihydroxyvitamin D3 J. Steroid. Biochem. Mol. Biol. 1995, 53, 599-602.

21. Lomtadze M. L., Khochava M. A., Shalamberidze. I. A., Shilakadze M. A., Dzhokhtaberidze T. G.: Functional status of haemostasis system in patients with visceral leishmaniasis. Georgian Med. News. 2005, 128, 59-62.

22. López-Peña M., Alemañ N., Muñoz F., Fondevila D., Suárez M. L., Goicoa A., Nieto J. M.: Visceral leishmaniasis with cardiac involvement in a dog: a case report. Acta Vet. Scand. 2009, 51, 20.

23. Mattner F., Smiroldo S., Galbiati F., Muller M., Di Lucia P., Poliani P. L., Adorini $L$.: Inhibition of Th1 development and treatment of chronic-relapsing experimental allergic encephalomyelitis by a non-hypercalcemic analogue of 1, 25-dihydroxyvitamin D3. Eur. J. Immunol. 2000, 30, 498-508.

24. Özata F., Ural K.: Thrombocyte indices in dogs infected with Ehrlichia canis and Anaplasma phagocytophilum. Rev. MVZ Cordoba 2014, 19, 4277-4288.

25. Ramos-Martínez E., Gutierrez-Kobeh L., Villaseñor-Cardoso M. I.: The role of vitamin D in the control of Leishmania infection. Can. J. Physiol. Pharmacol. 2015, 93, 369-376.

26. Rosa F. A., Leite J., Braga E. T., Moreira P., Baltazar F. H., Biondo A. W., Machado G. F.: Cardiac lesions in 30 dogs naturally infected with Leishmania infantum chagasi. Vet. Pathol. 2014, 51, 603-606.

27. Sato E., Imafuku S., Ishii K., Itoh R., Chou B., Soejima T., Hiromatsu K. Vitamin D-dependent cathelicidin inhibits Mycobacterium marinum infection in human monocytic cells. J. Dermatol. Sci. 2013, 70, 166-172.

28. Shor R., Tirosh A., Shemesh L., Krakover R., Bar Chaim A., Mor A.: 25 hydroxyvitamin $\mathrm{D}$ levels in patients undergoing coronary artery catheterization. Eur. J. Intern. Med. 2012, 23, 470-473.

29. Solano-Gallego L., Miró G., Koutinas A., Cardoso L., Pennisi M. G., Ferrer L., Baneth $G$.: LeishVet guidelines for the practical management of canine leishmaniosis. Parasit. Vectors. 2011, 4, 86.

30. Syal S. K., Kapoor A., Bhatia E., Sinha A., Kumar S., Tewari S.: Vitamin D deficiency, coronary artery disease, and endothelial dysfunction: observations from a coronary angiographic study in Indian patients. J. Inv. Cardiol. 2012, 24, 385-389.

31. Terrazzano G., Cortese L., Piantedosi D., Zappacosta S., Di Loria A., Santoro D., Ciaramella P.: Presence of anti-platelet IgM and $\mathrm{IgG}$ antibodies in dogs naturally infected by Leishmania infantum. Vet. Immunol. Immunopathol. 2006, 110, 331-337.

32. Torrent E., Leiva M., Segalés J., Franch J., Peña T., Cabrera B., Pastor J.: Myocarditis and generalised vasculitis associated with leishmaniosis in a dog. J. Small. Anim. Pract. 2005, 46, 549-552.

Corresponding author: Hasan Erdogan, DVM, PhD, Department of Internal Medicine, Faculty of Veterinary, Adnan Menderes University, Isikli, Aydın, 09017, Turkey; e-mail: hasan.erdogan@adu.edu.tr 